\title{
Theoretical Investigation on the Resonance Effect in the Acoustics
}

\author{
DU Chuan-mei \\ School of Civil Engineering and Architecture \\ Anhui University of Science and Technology \\ Huainan Anhui 232001, China \\ ducm1997@126.com
}

\author{
ZHANG Ming-xu \\ School of Civil Engineering and Architecture \\ Anhui University of Science and Technology \\ Huainan Anhui 232001, China
}

\author{
XU Ying \\ School of Civil Engineering and \\ Architecture \\ Anhui University of Science and \\ Technology \\ Huainan Anhui 232001, China
}

\author{
NI Xiu-quan \\ School of Civil Engineering and \\ Architecture \\ Anhui University of Science and \\ Technology \\ Huainan Anhui 232001, China \\ ducm1997@126.com
}

\author{
ZHOU Heng-linag \\ School of Civil Engineering and \\ Architecture \\ Anhui University of Science and \\ Technology \\ Huainan Anhui 232001, China
}

\begin{abstract}
In this paper, the resonance interaction at the acoustic vibration caused by the inharmonic vibration is discussed. Based on the perturbation theory, the influences of the resonance interaction between the two and three vibrant levels on the level energy, wave-function, and the spectral line intensity are investigated in detail respectively. The analytical expressions of the vibration level energy, wave-function, and spectral line intensity with the consideration of the resonance interaction are also obtained under the third order inharmonic approximation.
\end{abstract}

Keyword-Resonance; Vibration level; Wave-function; Spectral line intensity

\section{INTRODUCTION}

The analysis and application of acoustic resonance phenomenon had been researched. For example ,Sun Guangrong in the university of Nanking carried on a research responding to Steady State Frequency Response in roomsAcoustics of Room and Small Room to indoor steady state frequency ${ }^{[1]}$. Vera Markovi Etc has carried on theory analysis to the acoustic resonance ${ }^{[2-4]}$. The intensity and structural of vibrant acoustic spectrum take place to change according to the resonance interaction between the difference vibrant levels. The resonance interaction of the different vibrant levels causes spectral structure complicated and bring for difficulty for analysis of acoustic vibration structure. Such as figure 1 show:

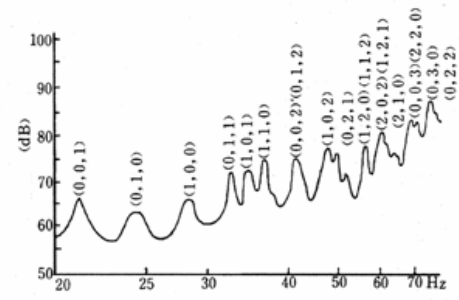

Fig.1. The actually measured resonance effect

Consequently, the theory study of the effect on acoustic spectrum structure with the consideration of the resonance interaction between the vibration levels have important instructional function for the correct analysis of the test of acoustic resonance spectrum .In this paper ,the basic model of the resonance interaction between the three vibration levels on the level energy, wave-function and the spectral line intensity are investigated in theory respectively and are also obtained the express of the theory investigated in detail .

\section{THE BASIC THEORY OF RESONANCE INTERACTION}

It is the most roughly approximation regarding vibration as harmonic vibration .It belongs to zero classes approximation .Actually, vibration is inharmonic . Therefore in motive Hamiltonian operator, higher order term of normal coordinate $Q_{i}$ should be considered for potential energy operator. In order to simplify the theory calculation , suppose in these harmonic vibration model $Q_{1} 、 Q_{2}$ and their overtones vibration have homological symmetry and vibrant frequency satisfy $v_{1} \approx 2 v_{2}$. The potential energy operator is 
evolved to three cubed of normal coordinate under the three level inharmonic approximation .Its expression:

$$
V=u_{e}+\frac{1}{2} \sum_{k} \lambda_{k} Q_{k}^{2}+\sum_{i, j, k} \alpha_{i, j, k} Q_{i} Q_{j} Q_{k}
$$

Then at this time vibration Hamiltonian can write for:

$$
\hat{H}=\hat{H}_{0}+\hat{H}^{\prime}
$$

Among which

$$
\begin{gathered}
\hat{H}_{0}=-\frac{\eta^{2}}{2} \sum \frac{\partial^{2}}{\partial Q_{k}^{2}}+\mu_{e}+\frac{1}{2} \sum_{k} \lambda_{k} Q_{k}^{2} \\
\hat{H}^{\prime}=\sum_{i j k} \alpha_{i j k} Q_{i} Q_{j} Q_{k} \\
=\alpha_{1,1,1} Q_{1}^{3}+\alpha_{1,1,2} Q_{1}^{2} Q_{2}+\alpha_{1,2,2} Q_{1} Q_{2}^{2}+\alpha_{2,2,2} Q_{2}^{3}
\end{gathered}
$$

$\alpha_{i j k}$ is three ranks inharmonic coefficient , the higher rank number is ,the more small inharmonic coefficient is. The $\mathrm{H}^{\prime}$ is a resonance function item, can be regarded as a perturbation item, be adopted as perturbation theory processing, We adopt three ranks inharmonic approximation in concrete calculation. Considering the resonance interaction, state function is the linear addition of the zero function with interaction of vibration state and keep same alike symmetry with the zero function, but make interaction of level energy take place to change, for $\hat{H}_{0}$ there have

$$
\hat{H}_{0} \psi_{n}^{(0)}=E_{n}^{(0)} \psi_{n}^{(0)}
$$

Then, considering resonance interaction there have

$$
\left(\hat{H}_{0}+\hat{H}^{\prime}\right) \psi=E \psi
$$

For solving the equation (6), will launch $\psi$ to the liner combining of zero class state function, then

$$
\psi=\sum_{n} a_{n} \psi_{n}^{(0)}
$$

got by the $(5,6,7)$

$$
\sum_{n}\left(E-E_{n}^{(0)}\right) a_{n} \psi_{n}^{(0)}-\hat{H}^{\prime} \sum_{n} a_{n} \psi_{n}^{(0)}=0
$$

The equation (8) both sides is left to multiply $\psi_{m}^{(0) *}$ and integral calculus, can get

$$
\sum_{n}\left[H_{m n}^{\prime}-\left(E-E_{m}^{(0)}\right) \delta_{m n}\right] a_{n}=0
$$

$$
\operatorname{det}\left|H_{m n}^{\prime}-\left(E-E_{m}^{(0)}\right) \delta_{m n}\right|=0
$$

Among which $\hat{H}_{m n}^{\prime}$ is the resonance interaction matrix element , from(9) and(10) can get the energy of vibration levels $E_{1}, E_{2}, E_{3} \Lambda$ and state function $\psi_{1}, \psi_{2}, \psi_{3}, \ldots \ldots$.

III.

\section{RESONANCE INTERACTIONS BETWEEN TWO VIBRATION} STATE

\section{A energy level and state function after the interaction}

Suppose the state function of vibration energy level $\left(V_{1}, V_{2}, V_{3}\right)$ is $\psi_{1}$, the state function of vibration energy level $\left(V_{1}+1, V_{2}-2, V_{3}\right)$ is $\psi_{2}$, then these two vibrations energy levels are almost degenerate, by equation (10) can get :

$$
\left|\begin{array}{cc}
E_{1}^{0}+H_{11}^{\prime}-E & H_{12}^{\prime} \\
H_{12}^{\prime} & E_{2}^{0}+H_{22}^{\prime}-E
\end{array}\right|=0
$$

Under three ranks inharmonic approximation there have:

$$
\begin{aligned}
& \hat{H}_{11}^{\prime}=H_{22}^{\prime}=0 \\
& H_{12}^{\prime}=\left\langle V_{1} V_{2} V_{3}\left|H^{\prime}\right| V_{1}+1, V_{2}-2, V_{3}\right\rangle \\
& =\alpha_{122}\left\langle V_{1} V_{2} V_{3}\left|Q_{1} Q_{2}^{2}\right| V_{1}+1, V_{2}-2, V_{3}\right\rangle \\
& =\alpha_{122}\left(\frac{1}{2 a}\right)^{3 / 2} \sqrt{\left(V_{1}+1\right)\left(V_{2}-1\right) V_{2}}
\end{aligned}
$$

In equation $a=\frac{4 \pi^{2} v_{e} \mu}{\eta}, v_{e}$ is vibrant frequency, $\mu$ is reduced mass, $\alpha_{122}=\frac{1}{6}\left(\frac{\partial^{3} U}{\partial Q_{1} \partial Q_{2}^{2}}\right)$ is three ranks inharmonic coefficient, from equation (11-13) can get:

$$
E_{ \pm}=\frac{1}{2}\left(E_{1}^{0}+E_{2}^{0}\right) \pm \frac{1}{2} \sqrt{\left(E_{1}^{0}+E_{2}^{0}\right)+4\left|H_{12}^{\prime}\right|^{2}}
$$

Thus get the abruption value of energy level:

$$
W_{\chi}=\frac{1}{2}\left(\sqrt{4\left|H_{12}^{\prime}\right|^{2}+\Delta_{0}^{2}}-\Delta_{0}\right), \quad \chi=1,2
$$

Among which $\Delta_{0}$ Is difference between $E_{1}^{o}$ and $E_{2}^{o}$ without considering perturbation, From equation (15) we can see the relation between energy level's splitting with resonance matrix element. The change of level energy is shown as below diagram considering interaction, among them $\Delta$ is difference between $E_{+}$and $\boldsymbol{E}_{-}$for considering perturbation. 


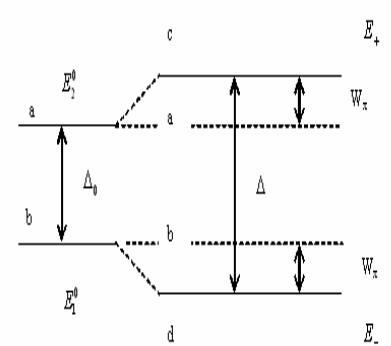

Fig.2. The influence of the resonance interaction on the energy of the two vibration levels

The state function of interaction can empress as linear combination of originally state function .

$$
\psi_{k}=a_{k} \psi_{1}^{o}+b_{k} \psi_{2,(k=1,2,)}^{0}
$$

Substitute the value of equation (14) into equation (9) can get the combination coefficient of state function respectively after

considering interaction .

$$
a_{k}=\left(\frac{H_{12}^{\prime 2}}{\left(E_{k}-E_{1}^{0}\right)^{2}+H_{12}^{\prime 2}}\right)^{1 / 2} \quad b_{k}=\left(\frac{\left(E_{k}-E_{1}^{0}\right)^{2}}{\left(E_{k}-E_{1}^{0}\right)^{2}+H_{12}^{\prime 2}}\right)^{1 / 2} \quad(\mathrm{k}=1,2)
$$

Then there have

$$
\frac{a_{k}}{b_{k}}=\left|\frac{H_{12}^{\prime}}{E_{k}-E_{1}^{0}}\right|
$$

Through reasonable approximation there have

$$
\frac{a_{k}}{b_{k}}=\left|\frac{\left(E_{1}^{0}+E_{2}^{0}\right) H_{12}^{\prime}}{E_{k}^{0}\left(E_{2}^{0}+E_{1}^{0}\right)+H_{12}^{\prime 2}}\right|
$$

Under the general situation has

$$
H_{12}^{\prime 2}<E_{k}^{0}\left(E_{2}^{0}+E_{1}^{0}\right) \quad(\mathrm{k}=1,2)
$$

Can prove

$$
\frac{d}{d H_{12}^{\prime}}\left(\frac{a_{k}}{b_{k}}\right)>0
$$

Therefore, the more $H_{12}^{\prime}$ is, the proportion of $\psi_{1}^{(0)}$ in $\psi_{1}$ and $\psi_{2}$ increase .

B spectral line intensity considering interaction

Spectral line intensity taking no account of interaction is:

$$
I_{k}^{o} \propto\left|\int \varphi_{0} \varphi_{k}^{0} d \tau\right|^{2}
$$

Among them $\varphi_{0}$ is lower state for transition, $\varphi_{k}^{0}$ is upper state for transition, $k=1 、 2$, considering interaction spectral line intensity is:

$$
\begin{gathered}
I_{k} \propto\left|\int \varphi_{0} \varphi_{k} d \tau\right|^{2} \\
=\left|\int \varphi_{0}\left(a_{k} \varphi_{1}^{0}+b_{k} \varphi_{2}^{0}\right) d \tau\right|^{2} \\
=a_{k}^{2} I_{1}^{0}+b_{k}^{2} I_{2}^{0}+2 a_{k} b_{k} \sqrt{I_{1}^{0} I_{2}^{0}}
\end{gathered}
$$

$$
=\left(\frac{H_{12}^{\prime 2}}{\left(E_{k}-E_{1}^{0}\right)^{2}+H_{12}^{\prime 2}}\right) I_{1}^{0}+\left(\frac{\left(E_{k}-E_{1}^{0}\right)^{2}}{\left(E_{k}-E_{1}^{0}\right)^{2}+H_{12}^{\prime 2}}\right) I_{2}^{0}+\frac{2 H_{12}^{\prime}\left(E_{k}-E_{1}^{0}\right)}{\left(E_{k}-E_{1}^{0}\right)^{2}+H_{12}^{\prime 2}} \sqrt{I_{1}^{0} I_{2}^{0}}
$$

IV. THE INTERACTION OF THREE RESONANCE VIBRATION STATE

A Energy levels and state function after considering interaction

State function of vibration energy level $\left(V_{1}-1, V_{2}+2, V_{3}\right)$ is $\psi_{1}$, state function of vibration energy level $\left(V_{1}, V_{2}, V_{3}\right)$ is $\psi_{3}$, these three vibration energy levels are approximately combined and belong to identical homology of the symmetry ,from equation (10) there have

$\left\|\begin{array}{ccc}E_{1}^{0}+H_{11}^{\prime}-E & H_{12}^{\prime} & H_{13}^{\prime} \\ H_{12}^{\prime} & E_{2}^{0}+H_{22}^{\prime}-E & H_{23}^{\prime} \\ H_{13}^{\prime} & H_{23}^{\prime} & E_{3}^{0}+H_{33}^{\prime}-E\end{array}\right\|=0$

Under three ranks inharmonic approximation there have

$$
\begin{gathered}
H_{11}^{\prime}=H_{22}^{\prime}=H_{33}^{\prime}=0 \\
H_{12}^{\prime}=\left\langle V_{1}-1, V_{2}+2, V_{3}\left|H^{\prime}\right| V_{1} V_{2} V_{3}\right\rangle \\
=\alpha_{122}\left\langle V_{1}-1, V_{2}+2, V_{3}\left|Q_{1} Q_{2}^{2}\right| V_{1} V_{2} V_{3}\right\rangle \\
=\alpha_{122}\left(\frac{1}{2 a}\right)^{3 / 2} \sqrt{V_{1}\left(V_{2}+2\right) V_{2}} \\
H_{23}^{\prime}=\left\langle V_{1}, V_{2}, V_{3}\left|H^{\prime}\right| V_{1}+1, V_{2}-2, V_{3}\right\rangle \\
=\alpha_{122}\left\langle V_{1} V_{2} V_{3}\left|Q_{1} Q_{2}^{2}\right| V_{1}+1, V_{2}-2, V_{3}\right\rangle \\
=\alpha_{122}\left(\frac{1}{2 a}\right)^{3 / 2} \sqrt{\left(V_{1}+1\right)\left(V_{2}-1\right) V_{2}}
\end{gathered}
$$

$$
H_{13}^{\prime}=0
$$

After considering interaction from equation (24-28), we canget energy of the three approximately combined vibration 
energy level respectively:

$$
\begin{gathered}
E_{1}=\sqrt{\frac{-P}{3}}\left(-\cos \frac{\phi}{3}+\sqrt{3} \sin \phi\right) ; \\
E_{2}=2 \sqrt{\frac{-P}{3}}\left(-\cos \frac{\phi}{3}\right) ; \\
E_{3}=\sqrt{\frac{-P}{3}}\left(-\cos \frac{\phi}{3}-\sqrt{3} \sin \phi\right)
\end{gathered}
$$

Among which

$$
\begin{gathered}
\phi=\cos ^{-1}\left(\frac{-q}{2 \sqrt{-p^{3} / 27}}\right) \\
p=\frac{E_{1}^{0} E_{2}^{0}+E_{2}^{0} E_{3}^{0}+E_{1}^{0} E_{3}^{0}-E_{1}^{2_{1}^{0}-E_{2}^{2_{2}^{0}}-E^{2^{0}}-3 H_{12}^{\prime 2}-3 H_{23}^{\prime 2}}}{3}
\end{gathered}
$$

$$
\begin{aligned}
& q=\frac{1}{27}\left[3 E_{1}^{2_{1}^{0}} E_{2}^{0}+3 E^{20} E_{3}^{0}+3 E^{20}{ }_{2}^{0} E_{3}^{0}+3 E_{1}^{0} E_{2}^{2_{2}^{0}}+3 E^{2}{ }_{3}^{0} E_{1}^{0}+3 E^{20} E_{2}^{0}-12 E_{1}^{0} E_{2}^{0} E_{3}^{0}\right. \\
& \left.-9\left(H_{12}^{\prime 2}+H_{23}^{\prime 2}\right)\left(E_{1}^{0}+E_{2}^{0}+E_{3}^{0}\right)+27 E_{1}^{0} H_{23}^{\prime 2}+27 E_{3}^{0} H_{12}^{\prime 2}-2 E_{1}^{3^{0}}-2 E_{2}^{3^{0}}-2 E_{3}^{3_{3}^{0}}\right]
\end{aligned}
$$

The state function of interaction can empress as linercombination of originally state function $\varphi_{k}=a_{k} \varphi_{1}^{0}+b_{k} \varphi_{2}^{0}+c_{k} \varphi_{3}^{0}$. Here, $k=1,2,3$, Substitute the value of equation (29) into equation (9) can get the combination coefficient of state function respectively after considering interaction .

$$
\begin{gathered}
a_{k}=\left[\frac{\left(E_{k}-E_{3}^{0}\right)^{2} H_{12}^{\prime 2}}{\left(E_{k}-E_{3}^{0}\right)^{2} H_{12}^{\prime 2}+\left(E_{1}^{0}-E_{k}\right)^{2}\left(E_{3}^{0}-E_{k}\right)^{2}+\left(E_{1}^{0}-E_{k}\right)^{2} H_{23}^{\prime 2}}\right]^{1 / 2} \\
b_{k}=\left[\frac{\left(E_{1}^{0}-E_{k}\right)^{2}\left(E_{3}^{0}-E_{k}\right)^{2}}{\left(E_{k}-E_{3}^{0}\right)^{2} H_{12}^{\prime 2}+\left(E_{1}^{0}-E_{k}\right)^{2}\left(E_{3}^{0}-E_{k}\right)^{2}+\left(E_{1}^{0}-E_{k}\right)^{2} H_{23}^{\prime 2}}\right]^{1 / 2}
\end{gathered}
$$

$$
c_{k}=\left[\frac{\left(E_{1}^{0}-E_{k}\right)^{2} H_{23}^{\prime 2}}{\left(E_{k}-E_{3}^{0}\right)^{2} H_{12}^{\prime 2}+\left(E_{1}^{0}-E_{k}\right)^{2}\left(E_{3}^{0}-E_{k}\right)^{2}+\left(E_{1}^{0}-E_{k}\right)^{2} H_{23}^{\prime 2}}\right]^{1 / 2}
$$

A series of formula of above can simply write:

$$
\begin{gathered}
a_{k}=\left[\frac{m_{k}^{2} H_{12}^{\prime 2}}{m_{k}^{2} H_{12}^{\prime 2}+n_{k}^{2} m_{k}^{2}+n_{k}^{2} H_{23}^{\prime 2}}\right]^{1 / 2} ; \\
b_{k}=\left[\frac{m_{k}^{2} n_{k}^{2}}{m_{k}^{2} H_{12}^{\prime 2}+n_{k}^{2} m_{k}^{2}+n_{k}^{2} H_{23}^{\prime 2}}\right]^{1 / 2} ; \\
c_{k}=\left[\frac{n_{k}^{2} H_{23}^{\prime 2}}{m_{k}^{2} H_{12}^{\prime 2}+n_{k}^{2} m_{k}^{2}+n_{k}^{2} H_{23}^{\prime 2}}\right]^{1 / 2}
\end{gathered}
$$

Among which $m_{k}=E_{k}-E_{3}^{0} ; n_{k}=E_{1}^{0}-E_{k}$;

$$
k=1,2,3
$$

\section{$B$ spectral line intensity considering interaction}

Spectral line intensity taking no account of interaction is:

$$
I_{k}^{o} \propto\left|\int \varphi_{0} \varphi_{k}^{0} d \tau\right|^{2}
$$

Among them $\varphi_{0}$ is lower state for transition, $\varphi_{k}^{0}$ is upper state for transition, $k=1,2,3$, considering interaction spectral line intensity is:

$$
\begin{gathered}
I_{k} \propto\left|\int \varphi_{0} \varphi_{k} d \tau\right|^{2} \\
=\left|\int \varphi_{0}\left(a_{k} \varphi_{1}^{0}+b_{k} \varphi_{2}^{0}+c_{k} \varphi_{3}^{0}\right) d \tau\right|^{2} \\
=a_{k}^{2} I_{1}^{0}+b_{k}^{2} I_{2}^{0}+c_{k}^{2} I_{3}^{0}+2 a_{k} b_{k} \sqrt{I_{1}^{0} I_{2}^{0}}+2 a_{k} c_{k} \sqrt{I_{1}^{0} I_{3}^{0}}+2 b_{k} c_{k} \sqrt{I_{2}^{0} I_{3}^{0}}= \\
{\left[\frac{m_{k}^{2} H_{12}^{\prime 2}}{m_{k}^{2} H_{12}^{\prime 2}+n_{k}^{2} m_{k}^{2}+n_{k}^{2} H_{23}^{\prime 2}}\right] I_{1}^{0}+\left[\frac{m_{k}^{2} n_{k}^{2}}{m_{k}^{2} H_{12}^{\prime 2}+n_{k}^{2} m_{k}^{2}+n_{k}^{2} H_{23}^{\prime 2}}\right] I_{2}^{0}}
\end{gathered}
$$$$
+\left[\frac{n_{k}^{2} H_{23}^{\prime 2}}{m_{k}^{2} H_{12}^{\prime 2}+n_{k}^{2} m_{k}^{2}+n_{k}^{2} H_{23}^{\prime 2}}\right] I_{3}^{0}+\frac{2 m_{k}^{4} n_{k}^{2} H_{12}^{\prime 2}}{m_{k}^{2} H_{12}^{\prime 2}+m_{k}^{2} n_{k}^{2}+n_{k}^{2} H_{23}^{\prime 2}} \sqrt{I_{1}^{0} I_{2}^{0}}
$$$$
+\frac{2 m_{k}^{2} n_{k}^{2} H_{12}^{\prime 2} H_{23}^{\prime 2}}{m_{k}^{2} H_{12}^{\prime 2}+m_{k}^{2} n_{k}^{2}+n_{k}^{2} H_{23}^{\prime 2}} \sqrt{I_{1}^{0} I_{3}^{0}}+\frac{2 m_{k}^{2} n_{k}^{4} H_{23}^{\prime 2}}{m_{k}^{2} H_{12}^{\prime 2}+m_{k}^{2} n_{k}^{2}+n_{k}^{2} H_{23}^{\prime 2}} \sqrt{I_{2}^{0} I_{3}^{0}}
$$

Among which $m_{k}=E_{k}-E_{3}^{0}, \quad n_{k}=E_{1}^{0}-E_{k}$

$$
(k=1,2,3)
$$

\section{ACKNOWLEDGMENT}

The work described in this paper was fully supported by the Civil engineering provincial pilot project, Constructional industry plan project in Anhui in 2011 (Project No.2011YF04 ), financially supported by National Basic Research Program of China under Grant No. 2012CB214900, China national coal association project(No. MTK2011-450).

\section{REFERENCE}

[1] SUN Guang-rong. Steady State Frequency Response in roomsAcoustics of Room and Small Room[J]. Electricity voice technique, 2010,34 (4) : 4-6.

[2] Vera Markovi, Bratislav Milovanovi and Olivera Proni. Determination of complex resonant frequencies in rectangular and circular cylindrical rooms[J] .Applied Acoustics, 2000, 59(3): 265-274.

[3] L. M. Dorojkine, S. V. Boritko, G. N. Dorojkina, A. V. Fokin and I. A. Rozanov. The resonant character of the sensitivity of the acoustic wave sensors [J]. Sensors and Actuators B: Chemical, 2003,89(1-2) :15-20.

[4] Neves e Sousa and B.M. Gibbs. Low frequency impact sound transmission in dwellings through homogeneous concrete floors and floating floors[J]. Applied Acoustics, 2011, 72(4) :177-189 